\title{
Bacterial 2-haloacid dehalogenases: Structures and catalytic properties
}

\author{
Kenji Soda*, Tatsuo Kurihara, Ji-Quan Liu, Vincenzo Nardi-Dei, Chung Park, \\ Masaru Miyagi\# ${ }^{\#}$ Susumu Tsunasawa ${ }^{\#}$, and Nobuyoshi Esaki \\ Laboratory of Microbial Biochemistry, Institute for Chemical Research, Kyoto \\ University, Uji, Kyoto 611, Japan; \#Biotechnology Research Laboratories, \\ Takara Shuzo Co., Ltd., Otsu, Shiga 520-21, Japan \\ * To whom correspondence should be addressed
}

\begin{abstract}
Haloacid dehalogenases (2-haloacid halidohydrolase; EC class: 3.8.1.2) catalyze the hydrolytic dehalogenation of 2-haloalkanoic acids to produce the corresponding 2-hydroxyalkanoic acids. Four different groups of 2haloacid dehalogenases have been found in bacterial cells. The carboxylate group of Asp ${ }^{10}$ of L-2-haloacid dehalogenase acts as a nucleophile on the $\alpha$-carbon of L-2-haloalkanoic acid to form an ester intermediate, which is hydrolyzed to produce the corresponding 2-hydroxyalkanoic acid. In contrast, in the reaction of DL-2-haloacid dehalogenase (inversion type), a water molecule activated by the enzyme directly attacks the $\alpha$-carbon of the substrate. D-2-Haloacid dehalogenase shows sequence similarity to DL-2-haloacid dehalogenase (inversion type), suggesting that the reaction mechanism of D-2-haloacid dehalogenase is similar to that of DL-2-haloacid dehalogenase (inversion type). Only the DL-2-haloacid dehalogenase (retention type) reaction proceeds with retention of the $\mathrm{C}_{2}$-configuration of the substrate, and its reaction mechanism is probably different from those of other 2-haloacid dehalogenases.
\end{abstract}

\section{Introduction}

2-Haloacid dehalogenases (EC class: 3.8 .1 .2 ) catalyze the hydrolytic dehalogenation of 2-haloalkanoic acids to produce the corresponding 2-hydroxyalkanoic acids. They are

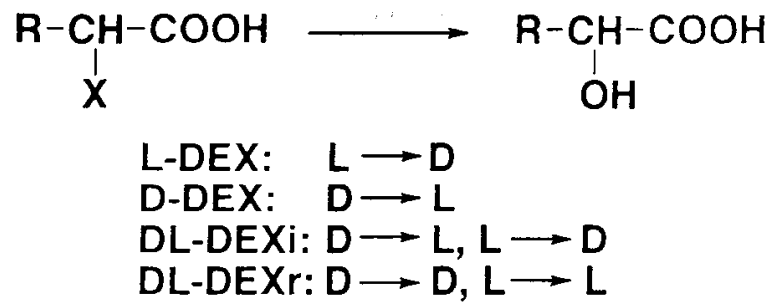

Fig. 1 Four types of 2-haloacid dehalogenases. 
classified into four groups based on their substrate and stereochemical specificities (Fig. 1). 1. L-2-Haloacid dehalogenase (L-DEX) specifically acts on L-2-haloalkanoic acids to produce the corresponding D-2-hydroxyalkanoic acids (1-7). 2. D-2-Haloacid dehalogenase (D-DEX) catalyzes the dehalogenation of D-2-haloalkanoic acids to the corresponding L-2hydroxyalkanoic acids $(7,8)$. 3. DL-2-Haloacid dehalogenase (inversion type) (DL-DEXi) dehalogenates both L- and D-2-haloalkanoic acids to the corresponding D- and L-2hydroxyalkanoic acids, respectively $(7,9,10)$. 4. DL-2-Haloacid dehalogenase (retention type) (DL-DEXr) acts on both L- and D-2-haloalkanoic acids to produce the corresponding Land D-2-hydroxyalkanoic acids, respectively (9).

Halogenated organic compounds are one of the largest groups of environmental pollutants, and accordingly dehalogenases that catalyze the degradation of these compounds attract a great deal of attention from the viewpoint of environmental technology. 2-Haloacid dehalogenases are also useful for the production of optically active 2-hydroxyalkanoic acids and 2-haloalkanoic acids, which are used as chiral synthons in chemical industry $(11,12)$. Relationship between structures and functions of 2-haloacid dehalogenases is interesting not only from the view point of basic enzymology, but also from the point of view of the molecular design of dehalogenases showing more efficient catalysis.

\section{L-2-Haloacid Dehalogenase}

L-DEXs have been isolated from various kinds of bacteria such as Pseudomonas (15), Xanthobacter (6), and Rhizobium species (7). The bacteria producing L-DEXs are more frequently isolated from the soil than those producing other types of 2-haloacid dehalogenases, when the screening is carried out using DL-2-chloropropionate as a sole carbon source (13). L-DEXs from various bacteria have several common properties. Their subunit molecular weights range from 25,000 to 28,000 , and they show the maximum reactivities in the $\mathrm{pH}$ range of 9-11. They specifically act on the L-isomer of a substrate. However, the subunit structures of L-DEXs are different from one another. they have structures of dimers $(3,5)$, monomers (2), and tetramers (4). Their substrate specificities are also different from one another with respect to the preferred carbon chain length of the substrates. L-2-Chloropropionate is better than chloroacetate as a substrate for L-DEX from Pseudomonas sp. YL (5), whereas the enzyme from Pseudomonas putida No. 109 prefers chloroacetate (2). Thermostabilities of these enzymes are different from one another as well. The enzyme of Pseudomonas sp. YL retains its full activity upon heating at $60^{\circ} \mathrm{C}$ for $30 \mathrm{~min}$, whereas the activity of the Pseudomonas putida No. 109 enzyme decreases to about $25 \%$ of the original activity.

The genes of L-DEXs from various bacteria have been cloned and analyzed. L-DEX degrades chloroacetate and bromoacetate, which are toxic for Escherichia coli. Accordingly, 
the L-DEX genes can be screened with chloroacetate or bromoacetate as a selection marker. Thus far, the primary structures of seven L-DEXs have been determined $(4,6,14-17)$. They show high similarities to one another (36-70\% identity). Haloacetate dehalogenase $\mathrm{H}-2$ from Moraxella sp. strain B (EC class: 3.8 .1 .3 ) is also significantly similar to these L-DEXs, although it specifically acts on haloacetates (18). The reactions of these dehalogenases probably proceed through the common mechanism, and their catalytic amino acid residues are supposed to be conserved among these enzymes. Recent computer analysis revealed that $\mathrm{L}$ DEXs belong to a large superfamily of hydrolases with diverse specificities including epoxide hydrolase and phosphoglycolate phosphatase. This suggests that these hydrolases catalyze the reaction through a similar mechanism (19).

L-DEX from Pseudomonas sp. YL has been studied in detail, and its reaction mechanism was proposed based on the comprehensive site-directed mutagenesis and $18_{0}$ incorporation experiments. All the 36 highly conserved charged and polar amino acid residues of L-DEX from Pseudomonas sp. YL, which consists of 232 amino acid residues, were replaced by other residues (20). The replacement of Asp ${ }^{10}, \mathrm{Asp}^{180}, \mathrm{Arg}^{41}, \mathrm{Lys}^{151}, \mathrm{Ser}^{175}$, $\mathrm{Thr}^{14}, \mathrm{Tyr}^{157}$, and Asn ${ }^{177}$ led to a significant loss of activity, and the replacement of Ser 118 caused a marked increase in the $K \mathrm{~m}$ value. Because replacement of these residues did not

(A)

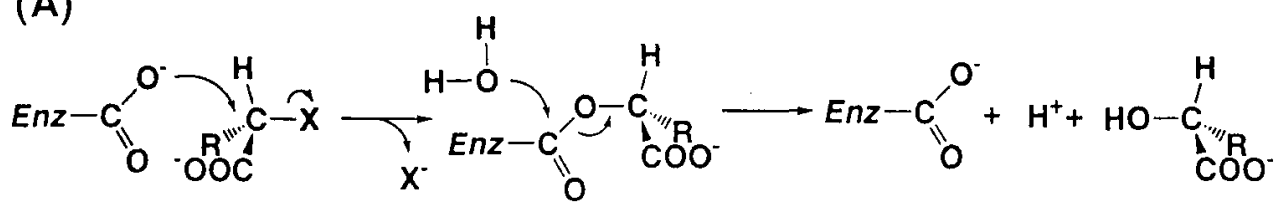

(B)

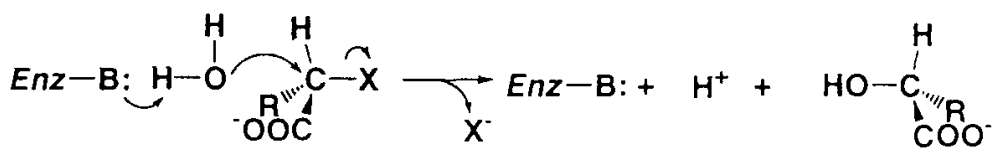

Fig. 2 Probable reaction mechanisms of 2-haloacid dehalogenases involving the inversion of the $\mathrm{C}_{2}$-configuration of the substrate.

cause a conformational change of the enzyme detectable on spectrophotometry and gel filtration, these residues are probably involved in catalysis.

Two different mechanisms have been proposed for the reactions of L-DEXs (Fig. 2). According to the Fig. 2A mechanism, a carboxylate group of the enzyme acts as a nucleophile to attack the $\alpha$-carbon of L-2-haloalkanoic acid, leading to the formation of an ester intermediate. This is hydrolyzed by an attack of the water molecule activated by a basic amino acid residue of the enzyme. Alternatively, water is activated by a catalytic base of the enzyme 
and directly attacks the $\alpha$-carbon of L-2-haloalkanoic acid to displace the halogen atom (Fig. 2B). We examined $18 \mathrm{O}$ incorporation experiment using $\mathrm{H}_{2} 18 \mathrm{O}$ in order to show which mechanism is involved in the reaction (21). When the multiple turnover enzyme reaction was carried out in $\mathrm{H}_{2}{ }^{18} \mathrm{O}$ with L-2-chloropropionate as a substrate and a large excess amount of the substrate, lactate produced was labeled with $18 \mathrm{O}$. However, when the single turnover enzyme reaction was carried out with a large excess amount of the enzyme, the product was not labeled. This suggests that an oxygen atom of the solvent water is first incorporated into the enzyme and then transferred to the product. This is consistent with the Fig. 2A mechanism, but not with the Fig. 2B mechanism. After the multiple turnover reaction in $\mathrm{H}_{2} 18 \mathrm{O}$, the enzyme was digested with lysyl endopeptidase, and the molecular masses of the peptide fragments formed were measured by an ionspray mass spectrometer. Two $18 \mathrm{O}$ atoms were shown to be incorporated into a hexapeptide, Gly6-Lys ${ }^{11}$. Tandem mass spectrometric analysis of this peptide revealed that $\mathrm{Asp}^{10}$ was labeled with two $18 \mathrm{O}$ atoms. These results indicate that Asp ${ }^{10}$ acts as a catalytic nucleophile, and thus the reaction proceeds through the Fig. 2A mechanism.

\section{D-2-Haloacid Dehalogenase}

The D-DEX reaction proceeds with inversion of the $\mathrm{C}_{2}$-configuration of the substrate forming L-2-hydroxyalkanoic acid. The enzyme has been purified to homogeneity from Pseudomonas putida strain AJ1/23 (8), and partially purified from Rhizobium species (7). The enzyme of Pseudomonas putida strain AJ1/23 has a molecular weight of about 135,000, and appears to be composed of four subunits of identical molecular weight. The maximum enzyme activity is found at $\mathrm{pH} 9.5$. The chain length of the substrate ranges from 2 to 4 . The subunit of the enzyme consists of 300 amino acid residues, and its molecular weight is 33,601 (22). The amino acid sequence of D-DEX shows no significant homology with those of LDEXs. The reaction mechanism of D-DEX is not clear.

\section{DL-2-Haloacid Dehalogenase (inversion type)}

DL-DEXi has been purified from Pseudomonas sp. 113 (10), and partially purified from Pseudomonas putida PP3 $(9,23)$ and Rhizobium species (7). The enzyme from Pseudomonas sp. 113 acts on 2-haloalkanoic acids whose carbon chain lengths are five or less. It also dehalogenates trichloroacetate to form oxalate and shows the maximum activity at pH9.5. The $K \mathrm{~m}$ values for substrates are as follows: $5.0 \mathrm{mM}$ for monochloroacetate, $1.1 \mathrm{mM}$ for L-2-chloropropionate, and $4.8 \mathrm{mM}$ for D-2-chloropropionate. The enzyme has a molecular weight of about 68,000 , and appears to be composed of two subunits identical in molecular weight. 
The gene encoding DL-DEXi of Pseudomonas sp. 113 was isolated, and its nucleotide sequence was determined (unpublished data). The amino acid sequence predicted shows about 20\% similarity to that of D-DEX of Pseudomonas putida strain AJ1/23. Accordingly, the dehalogenations of D-2-haloalkanoic acids catalyzed by DL-DEXi and D-DEX probably proceed through a similar mechanism.

The ${ }^{18} \mathrm{O}$ incorporation experiment with $\mathrm{H}_{2}{ }^{18} \mathrm{O}$ was carried out in the same way as for L-DEX of Pseudomonas sp. YL described above. When the single turnover enzyme reaction is carried out by use of a large excess amount of the enzyme, the product is labeled whether the substrate is $\mathrm{L}$ - or D-enantiomer (unpublished data). This suggests that an oxygen atom of the solvent water is directly incorporated into the product. Water molecule is probably activated by a catalytic base of the enzyme, and directly attacks the $\alpha$-carbon of D-and L-2-haloalkanoic acid to displace the halogen atom (Fig. 2B).

DL-DEXi is similar to L-DEX and D-DEX with respect to the substrate specificity, but it is not in the stereospecificity. All enzymes studied so far that work on the chiral carbon of substrates act on the exclusive enantiomers, except for the racemases, such as alanine racemase (24) and glutamate racemase (25). We are now studying whether the active site of DL-DEXi for D-enantiomers is identical with that for L-enantiomers.

\section{DL-2-Haloacid Dehalogenase (retention type)}

DL-DEXr acts on both D- and L-2-haloalkanoic acids as well as DL-DEXi. However, the DL-DEXr reaction proceeds with retention of the $\mathrm{C}_{2}$-configuration of the substrate. DLDEXr has been found only in Pseudomonas putida PP3 $(9,23)$. The enzyme catalyzes the hydrolytic cleavage of the carbon-halogen bond of 2-haloalkanoic acids with C2-C4. The enzyme is sensitive to SH-reagents, such as $N$-ethylmaleimide and $p$-chloromercuribenzoate, and the mechanism involving an active site cysteine residue is proposed: it involves a double inversion resulting in the retention of the $\mathrm{C}_{2}$-configuration of the substrate (Fig. 3). However, no direct evidence for this mechanism has been found. The sequence information is also not available.

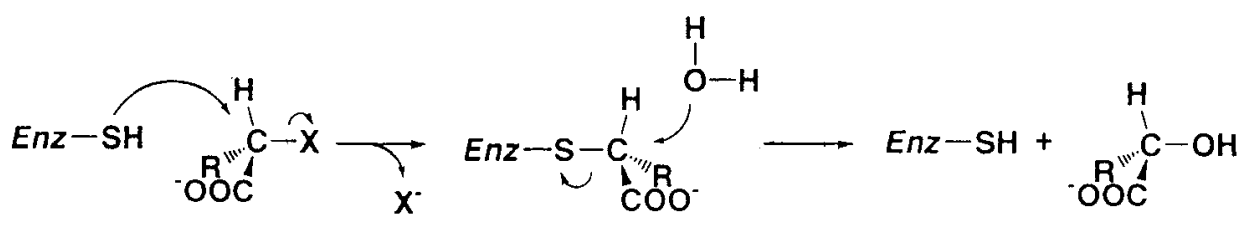

Fig. 3 Probable reaction mechanism of 2-haloacid dehalogenase involving the retention of the $\mathrm{C}_{2}$-configuration of the substrate. 


\section{Conclusion}

Four different kinds of 2-haloacid dehalogenases have been found in bacterial cells. The reaction mechanism of L-DEX has been studied most in detail to show the formation of ester intermediate of active site Asp ${ }^{10}$ with the substrate. The reaction mechanism of DL-DEXi is different from that of L-DEX in respect that the DL-DEXi reaction does not involve the enzyme-substrate ester intermediate. Instead, a water molecule is probably activated by a basic amino acid residue of DL-DEXi, and attacks the $\alpha$-carbon of the substrate. DL-DEXi is similar to D-DEX in the amino acid sequence. Thus, the dehalogenations of D-2-haloalkanoic acids catalyzed by both enzymes probably proceeds through a similar mechanism. Only the DLDEXr reaction proceeds with retention of the $\mathrm{C}_{2}$-configuration of the substrate. The reaction mechanism of this enzyme thus ought to be different from those of other 2-haloacid dehalogenases.

\section{REFERENCES}

1. P. Goldman, G. W. A. Milne \& D. B. Keister J. Biol. Chem. 243, 428-434 (1968).

2. K. Motosugi, N. Esaki \& K. Soda Agric. Biol. Chem. 46, 837-838 (1982).

3. J. S. H. Tsang, P. J. Sallis, A. T. Bull \& D. J. Hardman Arch. Microbiol. 150, 441446 (1988).

4. D. H. A. Jones, P. T. Barth, D. Byrom \& C. M. Thomas J. Gen. Microbiol. 138, 675-683 (1992)

5. J.-Q. Liu, T. Kurihara, A. K. M. Q. Hasan, V. Nardi-Dei, H. Koshikawa, N. Esaki \& K. Soda Appl. Environ. Microbiol. 60, 2389-2393 (1994).

6. J. van der Ploeg, G. van Hall \& D. B. Janssen J. Bacteriol. 173, $7925-7933$ (1991).

7. J. A. Leigh, A. J. Skinner \& R. A. Cooper FEMS Microbiol. Lett. 49, 353-356 (1988).

8. J. M. Smith, K. Harrison \& J. Colby J. Gen. Microbiol. 136, 881-886 (1990).

9. A. J. Weightman, A. L. Weightman \& J. H. Slater J. Gen. Microbiol. 128, 17551762 (1982).

10. K. Motosugi, N. Esaki \& K. Soda J. Bacteriol. 150, 522-527 (1982).

11. K. Motosugi, N. Esaki \& K. Soda Biotech. Bioeng. 26, 805-806 (1984).

12. A. K. M. Q. Hasan, K. Motosugi, N. Esaki \& K. Soda J. Ferment. Bioeng. 72, 481482 (1991).

13. K. Motosugi, N. Esaki \& K. Soda Arch. Microbiol. 131, 179-183 (1982).

14. U. Murdiyatmo, W. Asmara, J. S. H. Tsang, A. J. Baines, A. T. Bull \& D. J. Hardman Biochem. J. 284, 87-93 (1992). 
15. B. Schneider, R. Muller, R. Frank \& F. Lingens J. Bacteriol. 173, 1530-1535 (1991).

16. H. Kawasaki, T. Toyama, T. Maeda, H. Nishino \& K. Tonomura Biosci. Biotech. Biochem. 58, 160-163 (1994).

17. V. Nardi-Dei, T. Kurihara, T. Okamura, J.-Q. Liu, H. Koshikawa, H. Ozaki, Y. Terashima, N. Esaki \& K. Soda Appl. Environ. Microbiol. 60, 3375-3380 (1994).

18. H. Kawasaki, K. Tsuda, I. Matsushita \& K. Tonomura J. Gen. Microbiol. 138, 1317-1323 (1992).

19. E. V. Koonin and R. L. Tatusov J. Mol. Biol. 244, 125-132 (1994).

20. T. Kurihara, J.-Q. Liu, V. Nardi-Dei, H. Koshikawa, N. Esaki \& K. Soda $J$. Biochem. 117, 1317-1322 (1995).

21. J.-Q. Liu, T. Kurihara, M. Miyagi, N. Esaki \& K. Soda J. Biol. Chem. 270, 18309 18312 (1995).

22. P. T. Barth, L. Bolton \& J. C. Thomson J. Bacteriol. 174, 2612-2619 (1992).

23. A. J. Weightman, J. H. Slater \& A. T. Bull FEMS Microbiol. Lett. 6, 231-234 (1979).

24. K. Tanizawa, A. Ohshima, A. Scheidegger, K. Inagaki, H. Tanaka \& K. Soda Biochemistry 27, 1311-1316 (1988).

25. S. Y. Choi, N. Esaki, T. Yoshimura \& K. Soda J. Biochem. 112, 139-142 (1992). 